\title{
The Application of Activity Recognition Algorithm Based on Three-Dimensional Accelerometer in Virtual Reality Field
}

\author{
QingZhu ${ }^{1}$, Kai Yang $^{2}$, a, ZhiqiangWang ${ }^{3}$ \\ ${ }^{1}$ School of Software, Beijing University of Technology, Beijing100024, China; \\ ${ }^{2}$ School of Software, Beijing University of Technology, Beijing100024, China; \\ ${ }^{3}$ School of Software, Beijing University of Technology, Beijing100024, China. \\ alskrat@sina.com
}

Keywords:real-time human motion analysis, tri-axial acceleration transducer, $\mathrm{HCl}$, virtual reality.

\begin{abstract}
This paper aims at the head mounted display (HMD) equipment based on smart phones, puts forward ainteractive mode in order to improve the immersion of virtual reality (VR) games and applications.Its core is a real-time humanmotion analysis algorithmbased ontri-axial acceleration (TA) sensor. This algorithm uses smart phone built-in sensor to obtain the real-time TA signal produced by human movement, after the processing and classification to the signal through the threshold value method to classify motion state of user, such as: stand, walk, run and jump. Then the results directly reflected in the virtual scene, make user do the same behavior in VR games and applications. The experimental results show that the algorithm can quickly and accurately determine some motions,ensures that the user can get higherimmersion in VR experiences.
\end{abstract}

\section{Introduction}

With the development and popularization of VR technology [1], the VR solution of usessmart phonesbuilt-in gyroscope sensor to track head movements and uses smart phone's screen as a 3d display, is accepted by the masses of users. But this VR solution only can track head movements, its cause the interaction way too single, greatly limits the VR experiences.

Using smart phone built-in sensor to obtain the real-time TA signal produced by human movement can increase the interact way in smart phone VR games and application, improve the user's immersion.

Traditionalalgorithm of using TA sensor to analysishuman motions is mainly using machine learning [2, 3],extracteigenvalues and eigenvectors after collecting a large amount of data, and using the eigenvalues and eigenvectors to classify the data. In the step of data preprocess, most of traditionalalgorithms using sliding window to record the whole motion process, this causedthe delay. Main application fields of this algorithm are physical activity consumption detection [4], health monitoring and fall detection [5]. In these fields'people major focus on quantity of motion and the veracity of motion states and do not have too much requirements for real-time performance.

In VR games and applications, if the processing time is more than 200ms, the user will feel significant delay and dizziness. It makes the data which get form TA sensor need to be processing and decision in time (less than 200ms).

This algorithm will analyze the signal vector magnitude (SVM) [6, 7] and the change of SVM (CSVM), extracts extreme value and period of each behavior, and set the threshold value to classify motion state of user. Because the application fields of this algorithm are VR games and application, the algorithm can get individual sports information in tutorial, and make a series of threshold value to each user. In this way the algorithm can exclude the influence formheight, weight, age and behavioral habits of different user, to increase the recognition accuracy. 


\section{Datacollection and Processing}

Datacollection.This paper use SAMSUNG GT-N7100 as the testing equipment. Before the testfix the phone in the VR helmet and worn on the headas shown in Fig. 1(a). Set the sampling rate as $60 \mathrm{~Hz}$, follow the program prompts as shown in Fig. 1(b) do the actions of walk, run, jump and stand.

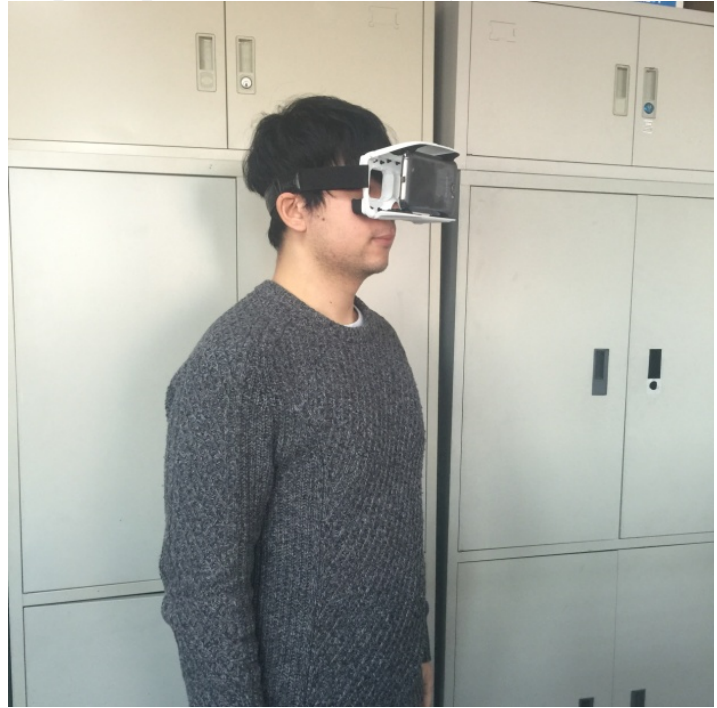

(a) Worn HMD

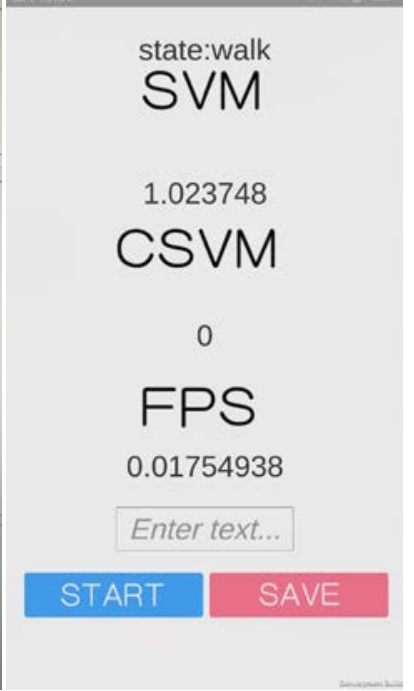

(b)Program interface

Fig. 1 Data collection

Data processing.The change of motion state, directly affects the movement of energy change, a lot of research shows that a linear correlation is found between themovement quantity of the body and human movement accelerationintegral for time [8]. Compare with $\mathrm{X}, \mathrm{Y}, \mathrm{Z}$ three axis acceleration data the SVM can show the movement of energy change clearer, and use SVM can reduce the vector computation complexity. At time t, X, Y, Z three axis instant accelerationisa $\mathrm{x}_{\mathrm{x}, \mathrm{t}}, \mathrm{a}_{\mathrm{y}, \mathrm{t}}, \mathrm{a}_{\mathrm{z}, \mathrm{t}}, \mathrm{SVM}_{\mathrm{isS}}$.

$$
\mathrm{S}_{\mathrm{t}}=\sqrt{\mathrm{a}_{\mathrm{x}, \mathrm{t}}^{2}+\mathrm{a}_{\mathrm{y}, \mathrm{t}}^{2}+\mathrm{a}_{\mathrm{z}, \mathrm{t}}^{2}}
$$

Graph of SVM is shown in Fig. 2. In order to get a better look of the speed of movement of energy change we also use CSVM data to analyze, in t time CSVM isC $\mathrm{t}_{\mathrm{t}}$.Graph of CSVM is shown in Fig. 3.To get the extreme value better, we using median filter to the SVM data the result is shown in Fig. 4

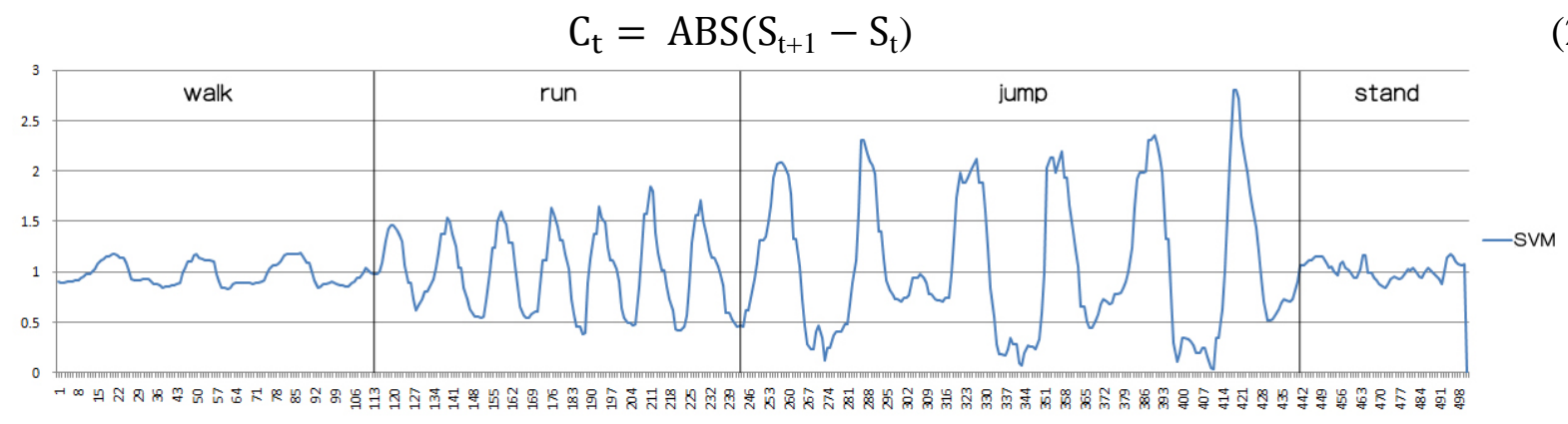

Fig. 2 The graph of SVM data

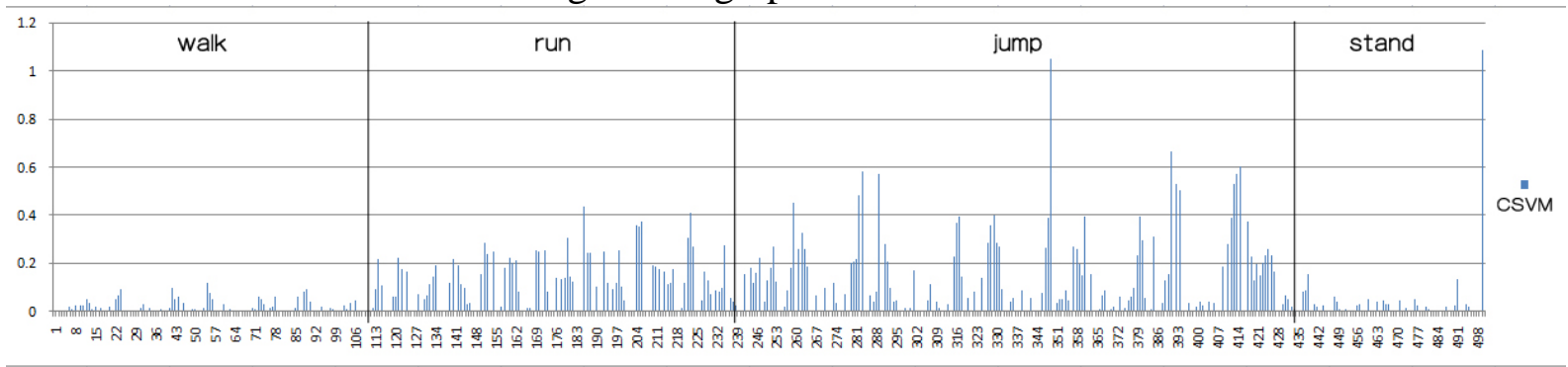

Fig. 3 The graph of CSVM data 


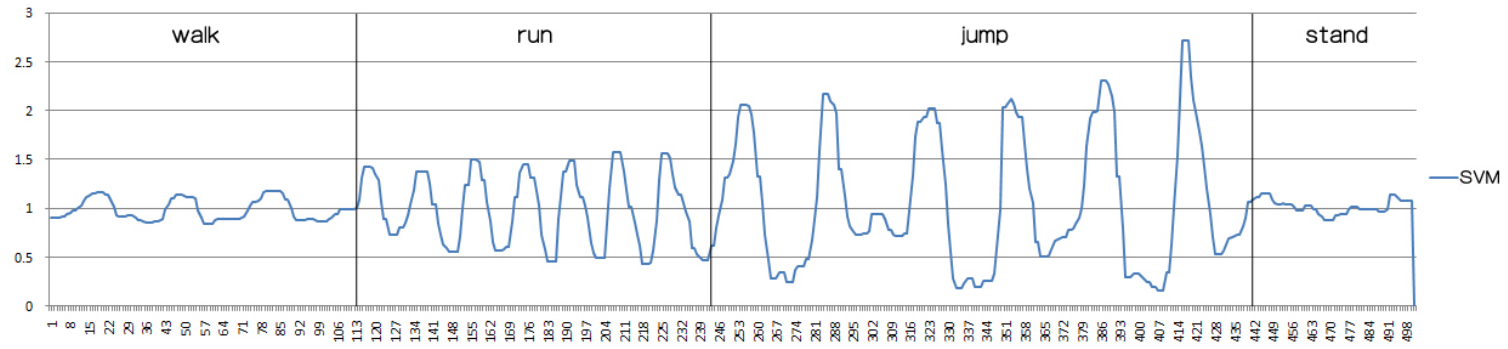

Fig. 4 The graph of SVM after filter

\section{Classification}

From test data analysis and comparison stand, walk, run, jump will be divided into two states. State1, low activity state, include stand and walk. State2, high activity state, include run and jump. Because of CSVM can show the change of movement of energy, and in thefirst time of switch state1 to state2, the change of energy is very evident, the maximum of CSVM in state1 $\mathrm{M}_{\text {low }}$ can be used as threshold value to distinguish state 1 and state2. Once appear $C_{i}$ is bigger than $M_{\text {low }}$ in the process of movement, the state1 will switch to state2.

$$
\mathrm{C}_{\mathrm{i}}>\mathrm{M}_{\text {low }} \rightarrow \text { State2 }
$$

But in state2, in one period of motion sometimes $C_{i}$ is smaller than $\mathrm{M}_{\text {low }}$, so we introduce the maximum of period of motion in state $2 T_{\text {high }}$, when there is $T_{\text {high }}$ consecutive $C_{i}$ is smaller than $M_{\text {low }}$, the state2 will switch to state1.

$$
\mathrm{C}_{\mathrm{n}}\left(\mathrm{n}=1,2, \ldots \mathrm{T}_{\text {high }}\right)<\mathrm{M}_{\text {low }} \rightarrow \text { State1 }
$$

To respectively distinguish stand and walk, run and jump, we use the maximum of SVM in running and standing, $S_{\operatorname{maxr}}$ and $S_{\operatorname{maxs}}$ as threshold value.

$$
\begin{aligned}
& \text { State } 1 \cap \mathrm{S}_{\mathrm{i}}<\mathrm{S}_{\text {maxs }} \rightarrow \text { Stand } \\
& \text { State } 1 \cap \mathrm{S}_{\mathrm{i}}>\mathrm{S}_{\text {maxs }} \rightarrow \text { Walk } \\
& \text { State } 2 \cap \mathrm{S}_{\mathrm{i}}<\mathrm{S}_{\text {maxr }} \rightarrow \text { Run } \\
& \text { State } 2 \cap \mathrm{S}_{\mathrm{i}}>\mathrm{S}_{\text {maxr }} \rightarrow \text { Jump }
\end{aligned}
$$

For four users, we ask them to do each action ten times and statistics the result is shown in Table 1.

Table 1 Experiment Results

\begin{tabular}{ccc}
\hline Action & Correct Number & Correct Rate \\
\hline Stand & 37 & $92.5 \%$ \\
Walk & 40 & $100 \%$ \\
Run & 38 & $95 \%$ \\
Jump & 35 & $87.5 \%$ \\
\hline
\end{tabular}

\section{Application in field of VR}

The interactive mode combines the algorithmin this paper and head movements tracking, can be applied in VR games and applications to get user much more agile control in VR experiences, to make the role in virtual world do the same action with user, then improve the user's immersion. Underrestrictions of the space of real world, sometimes user needs to do all the action on the spot, in this situation the mode in this paper also can bring a good experience to the users

\section{Summary}

This paperanalysis and applications the human movement data, come from the TA sensor. Building an interaction model looks on smartphone VR users, to increase the immersion successfully. Factual proof that in HCI field, interactive mode which more natural and genuine can increase the immersion. In the feedback of users many people point that the delay from state 2 switch state 1 is too long, and this algorithm doesn’t eliminate offset which come from the head move. 


\section{Acknowledgments}

This research is partially supported by Beijing Natural Science Foundation (Grant 4152008).

\section{References}

[1]Komura T, Lau R W H, Lin M C, et al. Virtual Reality Software and Technology[J]. Computer Graphics \& Applications IEEE, 2015, 35(5):20-21.

[2] Kwapisz J R, Weiss G M, Moore S A. Activity Recognition using Cell Phone Accelerometers[J]. AcmSigkdd Explorations Newsletter, 2010, 12(2):74-82.

[3] Khan A M, Lee $\mathrm{Y}$ K, Lee S Y, et al. Human Activity Recognition via an Accelerometer-Enabled-Smartphone Using Kernel Discriminant Analysis[C]// Future Information Technology (FutureTech), 2010 5th International Conference on. IEEE, 2010:1-6.

[4] Xie Y B N. RESEARCH AND APPLICATION OF PHYSICAL ACTIVITY CONSUMPTION DETECTION ON ANDROID SMART PHONE[J]. Computer Applications \& Software, 2012, 29(10):227-226.

[5] Na L I, Hou Y B, Huang Z Q. Implementation of a Real-time Fall Detection Algorithm Based on Body's Acceleration[J]. Journal of Chinese Computer Systems, 2012, 33(11):2410-2413.

[6] MATHIE Merryn J, COSTER Adelle C F, LOVELL Nigel H, et al. A pilot study of long term monitoring of human movements in the home using accelerometry $[\mathrm{J}]$. Journal of Telemedicine and Telecare, 2004, 10( 3) : 144-151.

[7] KARANTONIS Dean M, NARAYANAN Michael R, MATHIE Merryn, et al. Implementation of a real-time human movement classifier using a tri-axial accelerometer for ambulatory monitoring $[\mathrm{J}]$. IEEE Transactions on Information Technology in Biomedicine, 2006, 10 ( 1 ) : 156-167.

[8] Carlijn V C Bouten, Karel T M Koekkoek, Maarten Verduin, et al . A Triaxial Accelerometer and Portable DataProcessing Unit for the Assessment of Daily Physical Activity [J]. IEEE transactions on biomedical engineering, 1997( 3) . 